
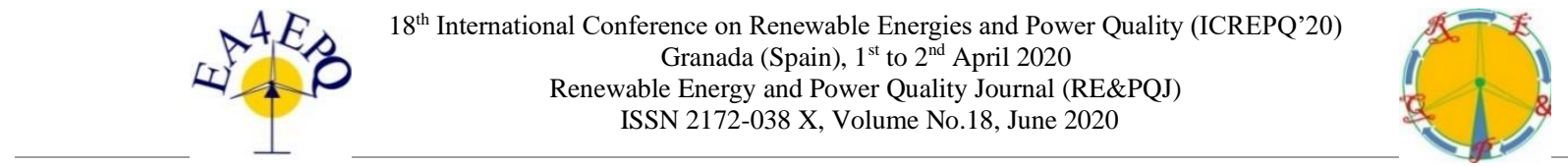

\title{
Estimation of Weibull parameters in winds speed mixture using non- linear optimization for wind energy applications
}

\author{
Francisco M. Arrabal-Campos ${ }^{1}$, Francisco G. Montoya ${ }^{1}$, Alfredo Alcayde ${ }^{1}$, Raúl Baños ${ }^{1}$ and Juan \\ Martínez-Lao ${ }^{1}$ \\ ${ }^{1}$ Department of Engineering \\ E.S.I., University of Almeria \\ Carretera del sacramento s/n - La Cañada, 04120 Almería (Spain) \\ Phone/Fax number: +34950214501, e-mail: fmarrabal@ual.es, pagilm@ual.es, aalcayde@ual.es, rbanos@ual.es, \\ jml357@ual.es
}

\begin{abstract}
Climate change and global warming are problems need to be tackled on a priority basis. The greenhouse gas (GHG) emissions and air pollution must be reduced by $25 \%$ and $40 \%$ compared to 1990 levels in 2020 and a reduction between $80 \%$ and $95 \%$ by 2050 . To mitigate the GHG emissions, countries have adopted policies to use renewable energy sources. In the case of wind energy, the statistical analysis of wind data is a crucial stage for estimating the wind turbine energy output through the turbine performance. The Weibull distribution has been widely used in the recent years for describing the behavior of the wind speed and it can be treated as a probability density function. Herein, it is presented a new method for calculating the Weibull parameters of an infinity sum of Weibull distributions. This new method is based on a Hilbert space generated by scale and form factor as Fredholm integral. This new method is named Inversion of the Weibull Distribution in wind speed mixture (IWeD). The simulations results indicate that IWeD is adequate for estimating the Weibull parameters when the wind speed is composed of several Weibull distributions.
\end{abstract}

Key words. Weibull distribution, wind speed mixture, Wind energy, Inversion winds speed mixture.

\section{Introduction}

Global warming and climate change are problems that countries and, in general terms, the society must face [1-5]. The growing demand for fossil fuels and environmental concerns such as greenhouse gas (GHG) emissions and air pollution has raised attention to renewable resources available for attending the growing demand of energy. In 1997, the most industrialized countries pledged, in Kyoto, to execute a set of measures to reduce GHG emissions by at least $5 \%$ on average between 2008 and 2012 compared to 1990 , entering into legal force in 2005. In this aspect, the Kyoto protocol proposes efficiently consume oil and coal, adopting renewable forms of energy. Industrialized countries should reduce their GHG emissions by $25 \%$ and
$40 \%$ compared to 1990 levels in 2020 and a reduction between $80 \%$ and $95 \%$ by 2050 [6].

In order to reduce the dependence on fossil fuels for mitigating the GHG emissions, countries have adopted policies to use renewable energy sources. Energy plays an important role in economic development and well-being of the society. Wind energy is widely recognized as one of the most sustainable sources of electricity, with a potential energy generation up to 40 times the annual global electricity consumption [7]. The environmental policies in the European Union (EU) and many other countries favor the growth in the production of wind energy, being them important to study the wind properties and its potential in terms of electricity generation [8].

Wind power is converted through a turbine, where the kinetic energy contained in the airflow turns the rotor of an electric generator. The wind turbine power output $\left(P_{t}\right)$, depends on the probability density function (PDF) of wind speed $(v)$. The wind turbine power output $\left(P_{t}\right)$, therefore, can be expressed by

$$
\bar{P}_{t}=\int_{0}^{\infty} P_{t}(v) f(v) d v
$$

where $P_{t}(v)$ is the wind turbine power output as function of the wind speed $(v)$ and $f(v)$ is the PDF.

Wind power in every region must be evaluated considering two main features: the potential and the feasibility [9]. The evaluation of the wind resource, the speed distribution and the average wind power must be studied among other parameters. Normally, several distributions of wind power are mixed and can generate differences in energy for turbines at nearby locations [10]. The statistical methods calculate the probability of the wind speed, and therefore, it is possible to determine the potential of the wind power.

The statistical analysis of wind data is the most crucial step for estimating the PDF as $(\boldsymbol{v})$ function. This analysis determines the calculation of the available energy and the 
wind turbine performance, which have a significative influence on the investment profitability. There are a lot of PDF for describing the behavior of wind data. The commonly used are beta function, lognormal function, Rayleigh and Weibull function [11-12].

The most used probability density function (which describes the wind speed distribution) is the Weibull function [13-14]. This function is widely studied in other engineering areas, as well as medical, chemistry and physics. The wind conditions are such that considering a mixture of Weibull distribution is more suitable for the description in real world. Mixture Weibull distribution is more useful because it can also show heterogeneous wind regimes, in which there is evidence of multimodality [15$16]$.

There are several numerical methods that can estimate the parameters of the Weibull distribution such as maximum likelihood estimation [17], least squares estimation [18], graphic method, moment method [19], power density method [20], among others.

The Weibull function is defined by the following parameters,

$$
F(v)=1-e^{-\left(\frac{v}{\lambda}\right)^{\kappa}}
$$

Where $\boldsymbol{F}(\boldsymbol{v})$ is the distribution function, $\kappa$ is the shape factor and $\lambda$ is the scaling factor. The parameters $\kappa$ and $\lambda$ are dimensionless parameters. The usual frequency distribution can be assumed and characterized by a shape factor of $\kappa=2$. In this case, this function is called Rayleigh function, which is a special distribution type of Weibull function. On real locations this parameter $\kappa$ may vary from 1.5 to 2.5 . For offshore locations, this parameter is about 1.5. Over land this parameter reaches values up to 2.5 or more depending on the specific location.

The Weibull distribution is defined by a probability density function, which is based on two factors named form factor $\kappa$ and scale factor $\lambda$, since is the derivative respect to wind speed of $\boldsymbol{F}(\boldsymbol{v})$. This probability density function is given by,

$$
f(v)=\frac{\kappa}{\lambda}\left(\frac{v}{\lambda}\right)^{\kappa-1} e^{-\left(\frac{v}{\lambda}\right)^{\kappa}}
$$

Where $\boldsymbol{f}(\boldsymbol{v})$ is the probability of measuring wind speed $\boldsymbol{v}$. This measurement of wind speed is performed at one height, but it is also possible to get an estimation at different heights [9].

The wind speed description is considered as a unique Weibull distribution. Sometimes, it is possible to take into account that the wind speed it is actually a sum of Weibull distributions. Equation (2) could be replaced by the following sum equation (3) that describes de probability of the wind speed.

$$
g(v)=\sum_{i=1}^{n} f(v)_{i}=\sum_{i=1}^{n}\left(\frac{\kappa_{i}}{\lambda_{i}}\right)\left(\frac{v}{\lambda_{i}}\right)^{\kappa_{i}-1} e^{-\left(\frac{v}{\lambda_{i}}\right)^{\kappa_{i}}}
$$

The sum of Weibull distributions can be derived as Fredholm integral. This integral equation has the form as follow,

$$
g(r)=\iint_{a}^{b} f(t, s) K(t, s, r) d t d s
$$

In this integral equation, $\boldsymbol{g}(\boldsymbol{r})$ and $\boldsymbol{K}(\boldsymbol{t}, \boldsymbol{s}, \boldsymbol{r})$ are known functions and the integration endpoints ' $a$ ' end ' $b$ ' are finite or infinite. Normally, $K$ is named kernel of the function, but it is a compact operator form a Banach space [21]. The goal is to solve for $f(t, s)$. For a continuous sum of Weibull distribution for any value of $\kappa$ and $\lambda, A(\kappa, \lambda)$, equation (3) could be replaced by the following integral equation (5) through the equation (4). Then, equation 3 is transformed to,

$$
g(v)=\iint_{0}^{\infty} A(\kappa, \lambda) \frac{\kappa}{\lambda}\left(\frac{v}{\lambda}\right)^{\kappa-1} e^{-\left(\frac{v}{\lambda}\right)^{\kappa}} d \kappa d \lambda
$$

Any value of $\kappa$ and $\lambda, A(\kappa, \lambda)$ is a matrix with the lengths of the two functions, which correspond with the parameters $\kappa$ and $\lambda$. The equation (5) could be also transformed into discrete space through the inner product of the $A(\kappa, \lambda)$ and the integral matrix of Weibull function. This discrete space is defined by the Hilbert space, which simplify the mathematical treatment. Equation (4) could be used as a linear algebraic form described by equation (4).

$$
g_{v}=H S \cdot A(\kappa, \lambda)
$$
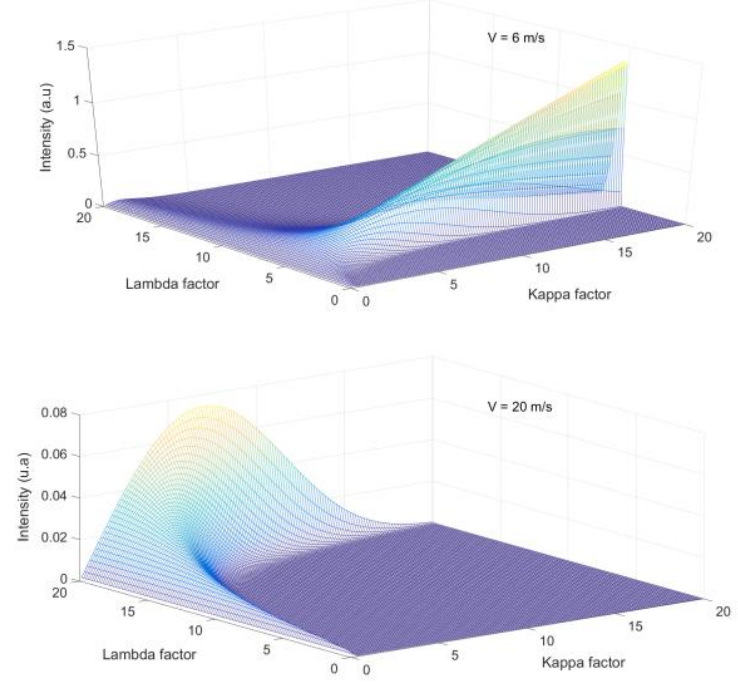

Fig. 1. Aspect of the Hilbert space for two different positions in the dimension $m$, which is determined by wind speed. Top is the position for wind speed equal to 6 and bottom is the position for wind speed equal to 20 .

where $\boldsymbol{g}_{\boldsymbol{v}}$ is equal to $\left[\boldsymbol{g}_{v \mathbf{1}}, \boldsymbol{g}_{\boldsymbol{v} \mathbf{2}}, \ldots, \boldsymbol{g}_{\boldsymbol{v} \boldsymbol{m}}\right]^{\mathrm{T}}$, the term A corresponds to a matrix of $n \times r$ elements of contribution of $\kappa$ and $\lambda$ factors, and HS, equation (8), is the $m \times n \times r$ matrix corresponding of the Hilbert space defined by equation (5), 


$$
H S_{m n r}=\frac{\kappa_{n}}{\lambda_{r}}\left(\frac{v_{m}}{\lambda_{r}}\right)^{\kappa_{n}-1} e^{-\left(\frac{v_{m}}{\lambda_{r}}\right)^{\kappa_{n}}}
$$

The equation (8) can be visualized in the Figure 1, where they are observed different positions of the dimension $m$ corresponding to wind speed. In this case, wind speed equal to 6 and equal to 20 is shown.

In general terms, the values of $A(\kappa, \lambda)$ can be estimated from experimental data and $g_{v}$ using the inversion of the equation (6). The discretization of Fredholm integral equations create discrete ill-posed problems. Therefore, this inversion is an ill-posed problem which was originally introduced by Hadamard [22]. Since the initial and boundary conditions are not well-defined, eventually strong vulnerability to noise and numerical instability have induced the emergence of different approaches. In order to face these problems, we can regard Levenberg-Marquardt statistical method [23] and SPLMOD [24]. All these approaches consider solutions with discrete values. Instead, other methods consider the solution as a distribution are CONTIN [25] maximum entropy (MaxEnt) [26] and more recently Proximal Algorithm for L1 combined with MaxEnt prior [27]. This problem can be solved using the widely known Tikhonov regularization method [28] for ill-posed problems and its formulation yield,

$$
\min \left\|H S \cdot A-g_{v}\right\|+\alpha f(A)
$$

$f(A)$ is the regularization function, and $\alpha$ balances the solution overfitting, such that the value of cost function is penalized on the basis of some prior assumptions. The value of $\alpha$ should be carefully chosen with respect to the noise level. The function of $f(A)$ imposes the solution form. Prior assumptions are included using $f(A)$, this function is represented with different forms,

$$
f(A)=\left\{\begin{array}{c}
\|L A\|_{2}^{2} \\
\sum\left(\frac{A_{i}}{\sum A_{i}}\right) \log \left(\frac{A_{i}}{\sum A_{i}}\right) \\
\|A\|_{1}
\end{array}\right.
$$

In this case, it is proposed the $\mathrm{L}_{1}$ norm of $A$ as regularization function. This approach endeavors on the recovery of sparse solutions by using the 11-norm penalty function. The minimum of the equation (8) can be found by using very robust iterative thresholding algorithms (ISTAs) [29].

This method based on 11-norm promotes sparsity of the solutions $A(\kappa, \lambda)$. This implies assumption that the number of components is smaller as possible. Normally, ISTA algorithm itself has a rate of convergence proportional to $1 / k$, where $k$ is el number of iterations. Nesterov's module is applied to the ISTA algorithm, as accelerated method for increasing up to $1 / k^{2}$ the rate of convergence [30-31]. This module was executed as the following sequence. Note that $\gamma_{k} \leq 0$.

$$
\lambda_{0}=0, \lambda_{k}=\frac{1+\sqrt{1+4 \lambda_{k-1}^{2}}}{2}, \gamma_{k}=\frac{1-\lambda_{k}}{\lambda_{k+1}}
$$

$$
A_{k+1}^{\prime}=\left(1-\gamma_{k}\right) A_{k+1}+\gamma_{k} A_{k}
$$

This algorithm is called fast iterative shrinkagethresholding algorithm (FISTA) [32]. This algorithm is derived from ISTAs algorithms.

In this work, we present the application of FISTA to decompose mixture of wind speed distributions.

\section{Methods}

The FISTA algorithm for decomposing wind speed mixture is evaluated in different simulations. This method is named Inversion of the Weibull Distribution in wind speed mixture (IWeD). The FISTA algorithm was implemented in MATLAB and all computations were performed on a windows 64-bit personal computer with an Intel i7-3770k @ $3.5 \mathrm{GHz}$ and $24 \mathrm{~GB}$ of memory. A detailed description, including the MATLAB code, can be obtained upon request.

Several simulated data sets where chosen to represent analytical situations. First, the set A consists of three speed wind Weibull distribution with scaled factor equal to 20 and three different kappa values $1.5\left(\kappa_{1}\right), 3\left(\kappa_{2}\right)$ and $12\left(\kappa_{3}\right)$. Second, the set B consists of three speed wind Weibull distribution with shape factor equal to 10 and three different lambda values $5\left(\lambda_{1}\right), 10\left(\lambda_{2}\right)$ and $15\left(\lambda_{3}\right)$. Finally, the last data set $\mathrm{C}$, consists of three speed wind Weibull distribution too. In this case, we have mixed both data set $\mathrm{A}$ and $\mathrm{B}$. We have varying the values of kappa factor and lambda factor. The values of kappa factor are $1.5\left(\kappa_{1}\right), 3$ $\left(\kappa_{2}\right)$ and $12\left(\kappa_{3}\right)$ and the values of lambda factor are $5\left(\lambda_{1}\right)$, $10\left(\lambda_{2}\right)$ and $15\left(\lambda_{3}\right)$.

Figure 2 presents the sum of speed wind Weibull distribution of the set $\mathrm{A}$ for the first simulation, while Figure 3 shows the results obtained for the simulated experiment A consisting in the sum of three Speed wind Weibull distribution with values $1.5,3$ and 12 for $\kappa_{1}, \kappa_{2}$ and $\kappa_{3}$, when the inversion of the Weibull distribution (IWeD) is applied.

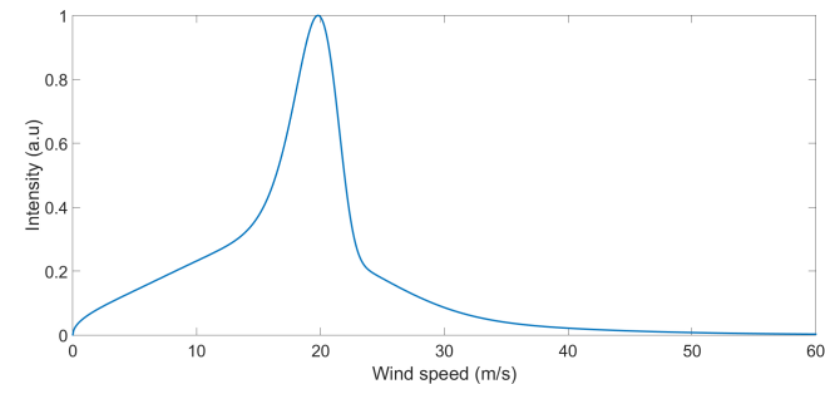

Fig. 2. Wind speed distribution composed by the sum of three Weibull distributions with scale factor equal to 20 and three different kappa values. These kappa values are 1.5, 3 and 12 .

For the second simulation, Figure 4 presents the sum of speed wind Weibull distribution for the set $\mathrm{B}$. The result obtained is shown in the figure 5 . This shows the results obtained on the simulated experiment B consisting in the 
sum of three wind speed Weibull distribution with values 5 , 10 and 15 for $\lambda_{1}, \lambda_{2}$ and $\lambda_{3}$, when it is applied the inversion of the Weibull distribution (IWeD).

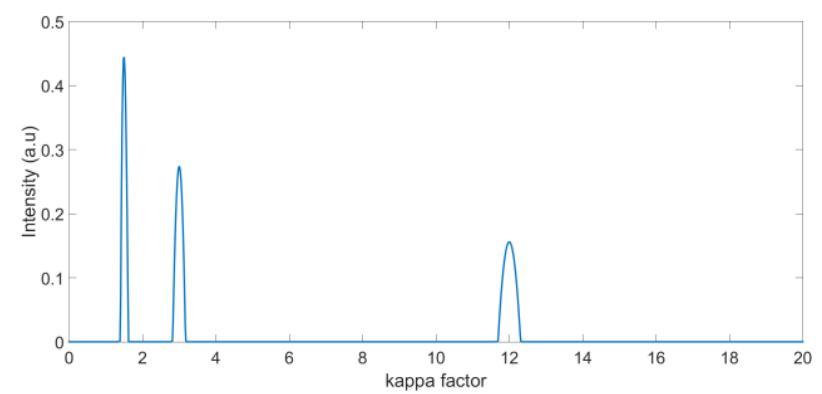

Fig. 3. Solution of the optimization release with FISTA algorithm. The kappa values detected are 1.5, 3 and 12 .

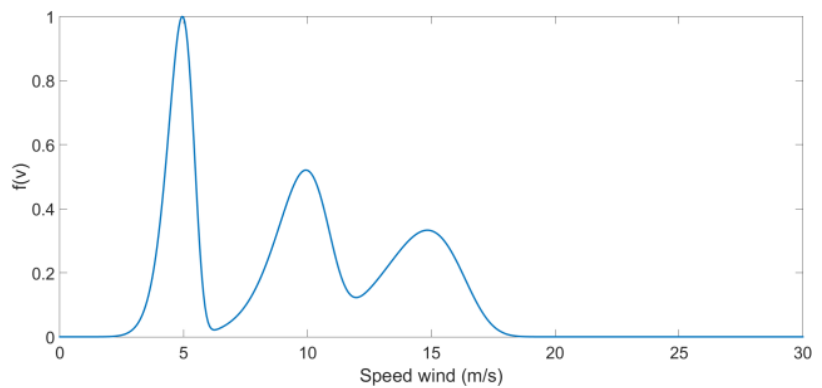

Fig. 4. Speed wind distribution composes by the sum of three Weibull distribution with shape factor equal to 10 and three different lambda values. These lambda values are 5, 10 and 15 .

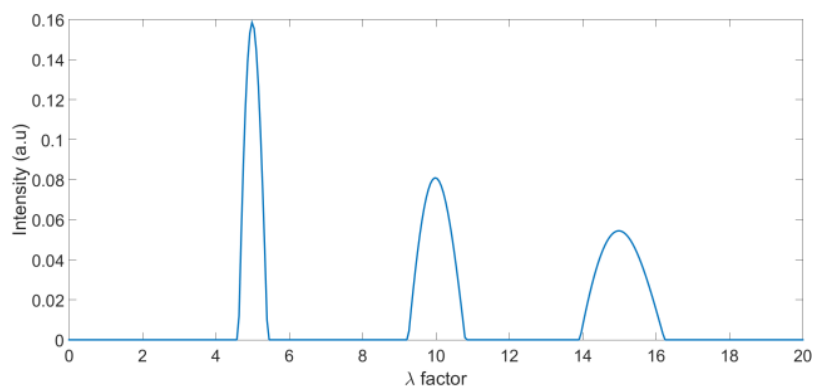

Fig. 5. Solution of the optimization release with FISTA algorithm. The lambda values detected are 5, 10 and 15 .

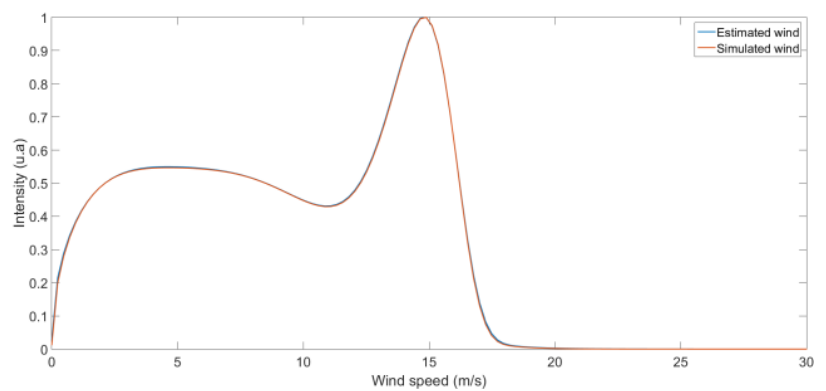

Fig. 6. Wind speed distribution composes by the sum of three Weibull distribution with three shape factors equal to 5, 10 and 15 and three different kappa values. These kappa values are 5, 10 and 15 .

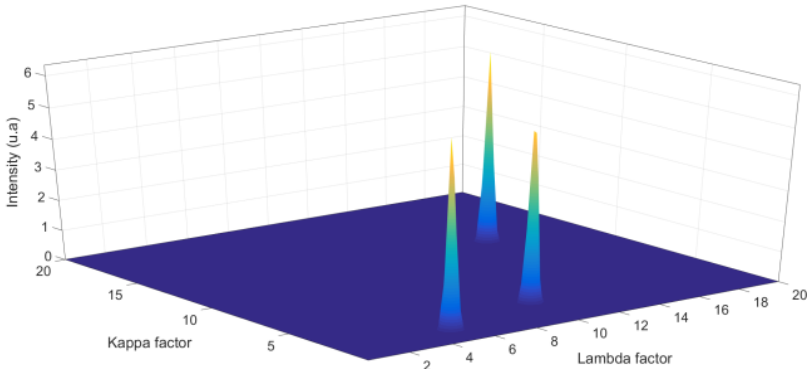

Fig. 7. Solution of the optimization release with FISTA algorithm. The lambda values detected are 5, 10 and 15. The kappa values detected are 1.5, 3 and 12 .

Figure 6 presents the sum of speed wind Weibull distribution for the last simulation, set C. Figure 7 shows the results obtained on the simulated experiment $\mathrm{C}$ consisting in the sum of three wind speed Weibull distribution with values $1.5\left(\kappa_{1}\right), 3\left(\kappa_{2}\right)$ and $12\left(\kappa_{3}\right)$ for kappa factor and 5, 10 and 15 for $\lambda_{1}, \lambda_{2}$ and $\lambda_{3}$, for lambda factor, when it is applied the inversion of the Weibull distribution (IWeD). Figure 7 presents the values of the matrix $A(\kappa, \lambda)$. The solution can be interpreted as follows: the solution has three different mixture of winds speed, where it is possible to get the coordinates for values of kappa and lambda factors. The combination of these wind speeds obtains a solution with coordinates equal to $(\kappa, \lambda)=\{(1.5,5),(3,10),(12,15)\}$.

\section{Conclusion}

Two and three-parameter Weibull distribution is still in development and it is probably on wind mixture where it can enhance understanding. In fact, this problem is solved for two winds mixed at most. This is crucial to understand the behavior of the wind and his theoretical power estimation. For these reasons, this paper presents the IWeD method for the analysis of winds speed mixture. The proposed method does need sparsity promote, which must be carefully chosen. This method is adequate for deconvolved winds speed mixtures. Three data sets A, B and $C$ have been simulated to show the potential of this approach, which is able to decompose the data sets mixture of wind speed distribution in its fundamental Weibull distributions. It is concluded that IWeD method is very suitable and efficient in order to estimate Weibull parameters for wind energy applications.

\section{Acknowledgement}

This work was supported by project PGC2018-098813- BC33 (Spanish "Ministerio de Ciencia, Innovación y Universidades"), and by European Regional Development Funds (ERDF).

\section{References}

[1] Zapata-Sierra, A.J., Cama-Pinto, A., Montoya, F.G., Alcayde, A., Manzano-Agugliaro, F. Wind missing data arrangement using wavelet-based techniques for getting maximum likelihood. Energy Conversion and Management 2019, 185, pp. 552-561. DOI: 10.1016/j.enconman.2019.01.109 
[2] Cama, A., Montoya, F.G., Gómez, J., De La Cruz, J.L., Manzano-Agugliaro, F. Integration of communication technologies in sensor networks to monitor the Amazon environment. Journal of Cleaner Production 2013, 59, pp. 32-42. DOI: 10.1016/j.jclepro.2013.06.041

[3] Al-mulali, U. Factors affecting $\mathrm{CO} 2$ emission in the Middle East: A panel data analysis. Energy 2012, 44 (1), pp. 564-569. DOI: 10.1016/j.energy.2012.05.045

[4] Al-mulali, U. Oil consumption, $\mathrm{CO} 2$ emission and economic growth in MENA countries. Energy 2011, 36 (10), pp. 6165-6171. DOI: 10.1016/j.energy.2011.07.048

[5] Sözen, A., Gülseven, Z., Arcaklioğlu, E. Forecasting based on sectoral energy consumption of GHGs in Turkey and mitigation policies. Energy Policy 2007, 35 (12), pp. 6491-6505. DOI: 10.1016/j.enpol.2007.08.024

[6] Kawase, R., Matsuoka, Y., Fujino, J. Decomposition analysis of $\mathrm{CO} 2$ emission in long-term climate stabilization scenarios. Energy Policy 2006, 34 (15), pp. 2113-2122. DOI: 10.1016/j.enpol.2005.02.005

[7] X. Lu and M. B. McElroy, "Global Potential for WindGenerated Electricity," in Wind Energy Engineering, vol. 106, no. 27, Elsevier, 2017, pp. 51-73.

[8] World Wind Energy Association. Press Release, February 2019. Wind turbines generate more than $1 \%$ of the global electricity; 2019. <http://www.wwindea.org> [accessed 22.10.19]

[9] A. Mostafaeipour, B. Bardel, K. Mohammadi, A. Sedaghat and Y. Dinpashoh, "Economic evaluation for cooling and ventilation of medicine storage warehouses utilizing wind catchers,", Renewable and Sustainable Energy Reviews (2014). Vol. 38, pp. 12-19.

[10] Mathew S., Philip G.S., Lim C.M., Analysis of Wind Regimes and Performance of Wind Turbines, Springer, Berlin, Heidelberg (2011).

[11] S. Mathew, Wind Energy. Berlin, Heidelberg: Springer Berlin Heidelberg, 2006.

[12] E. Hau, Wind Turbines. Berlin, Heidelberg: Springer Berlin Heidelberg, 2013.

[13] W. Weibull, "A Statistical Distribution Function of Wide Applicability”, Journal of Applied Mechanics (1959). pp. 293-297.

[14] K.E. Apt, "Applicability of the Weibull distribution function to atmospheric radioactivity data", Atmospheric Environment (1967). Vol. 10, pp. 777-781.

[15] P. Wais, "Two and three-parameter Weibull distribution in available wind power analysis," Renew. Energy, vol. 103, pp. 1529, 2017.

[16] J. A. Carta and P. Ramírez, "Analysis of two-component mixture Weibull statistics for estimation of wind speed distributions," Renew. Energy, vol. 32, no. 3, pp. 518-531, 2007.

[17] M.J.M. Stevens and P.T. Smulders, "The estimation of the parameters of the Weibull wind speed distribution for wind energy utilization purposes", Wind Eng (1979). Vol. 3, pp. 132-145.
[18] Y.K. Chu and J. Ke, "Computation approaches for parameter estimation of Weibull Distribution", Mathematical and Computational Applications (2012). Vol. 17, pp. 39-47.

[19] C.G. Justus, W.R. Hargraves, A. Mikhail, D. Graber, "Methods for estimating wind speed frequency distributions", J Appl Meteorol (1978). Vol. 17, pp. 350-353

[20] C.G. Justus, W.R. Hargraves, A. Mikhail and D. Graber, "Methods for estimating wind speed frequency distributions", J Appl Meteorol (1978). Vol. 17, pp. 350-353.

[21] A. Kirsch, An Introduction to the Mathematical Theory of Inverse Problems, vol. 120, no. 399. New York, NY: Springer New York, 2011.

[22] J. Hadamard, Lectures on the Cauchy Problem in Linear Partial Differential Equations, Yale University Press: New Haven, (1923).

[23] [1] K. Levenberg, "A method for the solution of certain nonlinear problems in least squares," Q. Appl. Math., vol. 2, no. 2, pp. 164-168, Jul. 1944.

[24] [1] S. W. Provencher, "A constrained regularization method for inverting data represented by linear algebraic or integral equations," Computer Physics Communications, vol. 27. pp. 213-227, 1982.

[25] [1] S. W. Provencher, "A constrained regularization method for inverting data represented by linear algebraic or integral equations," Comput. Phys. Commun., vol. 27, no. 3, pp. 213 227, Sep. 1982.

[26] S. F. Gull and J. Skilling, "Maximum entropy method in image processing," IEE Proc. F Commun. Radar Signal Process., vol. 131, no. 6, pp. 646-659, 1984.

[27]P. L. Combettes and J.-C. Pesquet, “A Douglas-Rachford Splitting Approach to Nonsmooth Convex Variational Signal Recovery,” IEEE J. Sel. Top. Signal Process., vol. 1, no. 4, pp. 564-574, Dec. 2007.

[28] A. N. Tikhonov, "Solution of incorrectly formulated problems and the regularization method", Soviet Math. Dokl. (1963). Vol. 4, 1035- 1038.

[29] C. R. Vogel and M. E. Oman, "Iterative methods for total variation denoising”, SIAM J. Sci. Comput. (1996). Vol. 17, pp. 227-238.

[30] Yu. E. Nesterov, "A method of solving a convex programming problem with convergence rate $\mathrm{O}(1 \mathrm{k} 2)$ ", Dokl. Akad. Nauk SSSR, 269:3 (1983), 543-547.

[31] Y. Nesterov, "Accelerating the cubic regularization of Newton's method on convex problems," Math Program., vol. 112, no. 1, pp. 159-181, Jul. 2007.

[32] A. Beck and M. Teboulle, SIAM J. Imaging Sci. (2009). Vol. 2, pp. 183-202. 